New windows on massive stars: asteroseismology, interferometry, and spectropolarimetry

Proceedings IAU Symposium No. 307, 2014

G. Meynet, C. Georgy, J. H. Groh 8 Ph. Stee, eds.

(C) International Astronomical Union 2015 doi:10.1017/S1743921314006528

\title{
Fundamental parameters of B type stars
}

\author{
María-Fernanda Nieva \\ Institute for Astro- and Particle Physics, Univ. of Innsbruck, Technikerstr. 25/8, 6020 \\ Innsbruck, Austria \\ Email: Maria-Fernanda.Nieva@uibk.ac.at
}

\begin{abstract}
Fundamental parameters of 26 well-studied sharp-lined single early B-type stars in OB associations and in the field within a distance of $\leqslant 400 \mathrm{pc}$ from the Sun are compared to high-precision data from detached eclipsing binaries (DEBs). Fundamental parameters are derived from accurate and precise atmospheric parameters determined earlier by us from non-LTE analyses of high-quality spectra, utilising the new Geneva stellar evolution models in the massrange $\sim 6$ to $18 M_{\odot}$ at metallicity $Z=0.014$. Evolutionary masses, radii and luminosities are determined to better than typically $5 \%, 10 \%$, and $20 \%$ uncertainty, respectively, facilitating the mass-radius and mass-luminosity relationships to be recovered for single core hydrogen-burning objects with a similar precision as derived from DEBs. Good agreement between evolutionary and spectroscopic masses is found. Absolute visual and bolometric magnitudes are derived to typically $\sim 0.15-0.20$ mag uncertainty. Metallicities are constrained to better than $15-20 \%$ uncertainty and tight constraints on evolutionary ages of the stars are provided. The spectroscopic distances and ages of individual sample stars agree with independently derived values for the host OB associations. The accuracy and precision achieved in the determination of fundamental stellar parameters from the quantitative spectroscopy of single early B-type stars comes close (within a factor 2-4) to data derived from DEBs.
\end{abstract}

Keywords. stars: early-type, stars: evolution, stars: fundamental parameters

\section{Introduction}

Comprehensive tests of stellar evolution models require as accurate characterisation of all stellar properties as possible. Besides atmospheric parameters like effective temperature $T_{\text {eff }}$ and surface gravity $\log g$ and elemental abundances, a knowledge of fundamental stellar parameters mass $M$, radius $R$ and luminosity $L$, and of age $\tau$ is necessary. Primary source of such data are double-lined detached eclipsing binaries (DEBs), which allow a direct determination of accurate masses and radii at a precision of $1-2 \%$. As

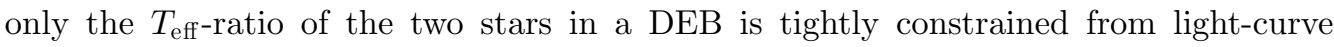
and radial-velocity-curve analysis, but not $T_{\text {eff }}$ of the components, stellar luminosities remain slightly less constrained. Ages need to be derived by comparison with theoretical isochrones, with the condition that both components have to be coeval. The most accurate and precise fundamental parameters for massive stars available at present can be found in the compilation by Torres et al. (2010) of early B-type stars.

However, the number of DEBs is limited, and it would be highly valuable if data of high quality could be obtained for single stars. The best candidate stars are located in clusters or associations, where distances (and therefore luminosities) and ages can be constrained from photometry and main-sequence fitting.

We have previously improved the modelling and analysis of the atmospheres of early B-stars by introducing non-LTE line-formation calculations based on a new generation of sophisticated model atoms (Nieva \& Przybilla 2006, 2007, 2008; Przybilla et al. 2008). Analyses of two samples of stars based on these models (Nieva \& Simón-Díaz 2011; 

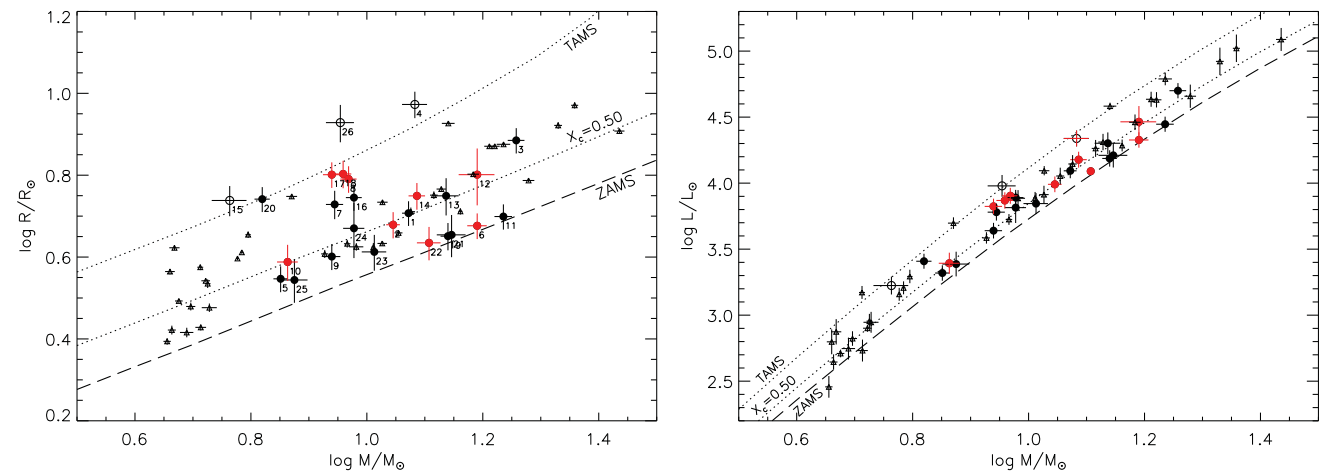

Figure 1. Mass-radius (left panel) and mass-luminosity (right panel) relationships for the sample stars. Black/red dots denote CN-unmixed/mixed chemical composition and open thick circles objects near/beyond core-H exhaustion. Wide circles surrounding the dots mark magnetic stars. Error bars denote $1 \sigma$-uncertainties. Data from DEBs (Torres et al. 2010) are shown for comparison (small triangles). Abscissa values are evolutionary masses. The ZAMS, 50\% core-H depletion and the TAMS are indicated by the dashed/thick/thin-dotted lines, as predicted by the stellar evolution models of Ekström et al. (2012).

Nieva \& Przybilla 2012), provide a highly accurate and precise atmospheric parameters and chemical abundances. Here, we discuss in particular the fundamental parameters $M, R, L$ of the stars (Nieva \& Przybilla 2014). As these are (effectively) single stars, stellar evolution models need to be employed in their derivation. We focus on the grids by Ekström et al. (2012), computed for the same metallicity as found for the early B-stars in the solar neighbourhood (Nieva \& Przybilla 2012), $Z=0.014$.

\section{Mass-radius and mass-luminosity relationships}

Our sample stars consist of 26 apparently slowly-rotating single early B-type stars in $\mathrm{OB}$ associations and in the field within a distance of $\leqslant 400 \mathrm{pc}$ from the Sun. We compare their mass-radius and mass-luminosity relationships with those deduced from DEBs in Fig. 1. We want to draw the attention to the extremely small error bars for these particular DEB data - both components of a DEB have masses and radii determined to $\pm 3 \%$, or better. These comprise only data of highest accuracy and precision discussed within the much broader literature on DEBs. Our sample stars fit well into the trends, with error bars coming close to that of the DEB components, typically within a factor $2-4$. The precision in luminosity even reaches similar values as that obtained for DEBs. This opens up the possibility to improve on the number statistics and in particular to trace the regions close to the ZAMS in the $M-R$ and $M-L$ relations for the more massive objects, which so far is not covered by DEBs.

\section{References}

Ekström, S., Georgy, C., Eggenberger, P., et al. 2012, A\&A A 537, A146

Nieva, M. F. \& Przybilla, N. 2006, ApJ (Letters) 639, L39

Nieva, M. F. \& Przybilla, N. 2007, A\&SA 467, 295

Nieva, M. F. \& Przybilla, N. 2008, A\&A 481, 199

Nieva, M.-F. \& Przybilla, N. 2012, A\& A 539, A143

Nieva, M.-F. \& Przybilla, N. 2014, A\&A 566, A7

Nieva, M.-F. \& Simón-Díaz, S. 2011, A\&A 532, A2

Przybilla, N., Nieva, M.-F., \& Butler, K. 2008, ApJ (Letters) 688, L103

Torres, G., Andersen, J., \& Giménez, A. 2010, A\&̊A Rev. 18, 67 